Benjamin Märkli

\title{
Die «Corona-Verordnung» des Bundesrats vom 28. Februar 2020
}

Der Beitrag untersucht die jüngst erlassene Verordnung über Massnahmen zur Bekämpfung des Coronavirus COVID-19, fasst ihren Inhalt zusammen und beurteilt verschiedene sich stellende Rechtsfragen. Er legt damit eine erste Auslegeordnung für die Betroffenen und die zum Vollzug zuständigen Kantone vor.

Beitragsart: Beiträge

Rechtsgebiete: Gesundheitsrecht 


\section{Inhaltsübersicht}

1. Allgemeines

2. Die Verordnung

2.1. Zusammenfassung des Inhalts

2.2. Rechtsgrundlage

3. Veranstaltungen mit mehr als 1000 Personen

3.1. Rechtsnatur

3.2. Erfasste Veranstaltungen

3.3. Verletzungen

4. Veranstaltungen mit weniger als 1000 Personen

4.1. Rechtsnatur

4.2. Erfasste Veranstaltungen

4.3. Rechtsfolgen

5. Rechtsschutz

6. Fazit

\section{Allgemeines}

[1] Die Corona-Verordnung ${ }^{1}$ (im Folgenden: COVO) ist am vorletzten Freitag, 28. Februar 2020 in Kraft getreten. Dass in der Zeit bis zur Publikation dieses Beitrags nicht mehr als eine erste Auslegeordnung möglich ist, versteht sich von selbst. Dennoch scheint eine erste wissenschaftliche Auseinandersetzung mit dem Inhalt der Verordnung notwendig, da sie in ihren Auswirkungen doch sehr einschneidend ist. Da der Bundesrat, beziehungsweise das Bundesamt für Gesundheit, die COVO zweifellos unter sehr grossem Zeitdruck ausarbeiten mussten, waren gewisse Unklarheiten in der Anwendung sowie ein zunächst uneinheitlicher Vollzug nachvollziehbar. Auch diesbezüglich versucht der Beitrag, Abhilfe zu schaffen.

[2] Die Verordnung ist dem Recht der besonderen und ausserordentlichen Lagen ${ }^{2}$ zuzurechnen. Die Schwierigkeit dabei ist einerseits die zeitliche Dringlichkeit, denn die notwendigen Anordnungen müssen schnell getroffen werden; andererseits bestehen Unklarheiten über die Faktenlage. ${ }^{3}$ Dies zeigt sich auch im vorliegenden Fall, da die Ausbreitung des Coronavirus rasant erfolgte, nach wie vor aber gewisse Unklarheiten über die Krankheit zu bestehen scheinen. Hinzu tritt, dass es sich um Massnahmen zur Prävention handelt. Die Grundproblematik bei der Prävention einer Krankheit ist, dass frühzeitig einschneidende Massnahmen ergriffen werden müssen, um eine weitere Ausbreitung möglichst zu verhindern. ${ }^{4}$ Dies steht in einem offensichtlichen Spannungsverhältnis zum Verhältnismässigkeitsgebot und führt zu einem Präventions-Paradoxon: Ist eine Massnahme geeignet, muss ihre Erforderlichkeit suspekt sein; ist sie klar erforderlich, dürfte ihr die Eignung fehlen. Zudem ist die Evaluation ex post schwierig, denn das Endergebnis ist bei einer unnötigen und einer erfolgreichen Prävention das gleiche, nämlich dass im Idealfall eben gerade keine Epidemie auftritt. ${ }^{5}$

Verordnung über Massnahmen zur Bekämpfung des Coronavirus (COVID-19) vom 28. Februar 2020 (SR 818.101.24).

2 Zu den Begriffen David Rechsteiner, Recht in besonderen und ausserordentlichen Lagen, St. Gallen 2016, Rn. 6 ff.

3 BGE 132 II 305 E. 4.4 S. 321 ff.; Rechsteiner (Fn. 2), Rn. 66.

4 Thomas Gächter/Bernhard Rütsche, Gesundheitsrecht, 4. Aufl., Basel 2018, Rn. 777.

5 Vgl. auch Gä́hter/Rütsche (Fn. 4), Rn. 780. 
[3] Anlass für die COVO ist die rasche Ausbreitung des neuen Coronavirus, das Ende 2019 in der chinesischen Stadt Wuhan entdeckt wurde. Nach Angaben des Bundesamts für Gesundheit ${ }^{6}$ gehört das Coronavirus «SARS-CoV-2» zur gleichen Familie wie die Erreger MERS und SARS. Die durch das neue Coronavirus verursachte Krankheit wird als «Coronavirus-Krankheit 2019» bzw. «COVID-19» bezeichnet.

[4] Übertragen werde das neue Coronavirus «hauptsächlich bei engem und längerem Kontakt». Ein solcher besteht «bei weniger als 2 Metern Abstand während mehr als 15 Minuten», wie das Bundesamt für Gesundheit auf seiner Webseite schreibt. Die eigentliche Übertragung des Coronavirus erfolgt durch Tröpfchen. Dabei überleben die ausgeschiedenen Viren «einige Stunden in winzigen Tröpfchen auf Händen oder Oberflächen». Ob man sich nach einer Berührung solcher Oberflächen über die Schleimhäute anstecken kann, sei zurzeit nicht klar. ${ }^{7}$

\section{Die Verordnung}

\subsection{Zusammenfassung des Inhalts}

[5] Art. 1 COVO ist eine Zwecknorm. Sie ist ohne eigene Tragweite, dient aber bei der Auslegung der übrigen Teile der Verordnung als Leitlinie. Einschlägig ist ausschliesslich Art. 1 Abs. 2 COVO, da nur er sich zum Zweck äussert; er bewegt sich im Rahmen von Art. 1 Abs. 2 Bst. f EpG ${ }^{8}$. Art. 1 Abs. 1 COVO ist demgegenüber bedeutungslos, denn die Anordnung von Massnahmen ergibt sich ohne Weiteres aus dem Rest der Verordnung. Das blosse Anordnen von Massnahmen wäre auch kein zulässiger Zweck, sondern ist nur ein Mittel, mit dem ein Zweck erreicht werden soll.

[6] Das Herzstück der COVO bildet Art. 2, der ein schweizweites Versammlungsverbot für Versammlungen mit mehr als 1000 Personen enthält und für kleinere Versammlungen vorsieht, dass die Veranstalterinnen und Veranstalter in Absprache «mit der zuständigen kantonalen Behörde» eine Risikoabwägung vornehmen müssen. Das Veranstaltungsverbot ist befristet bis zum 15. März 2020.

[7] Art. 3 COVO verschafft den kantonalen Behörden umfassende Zutritts- und Kontrollrechte und verpflichtet die Veranstalterinnen und Veranstalter, den Anordnungen der Behörden unverzüglich Folge zu leisten. Damit ist die Bestimmung im Zusammenhang mit Art. 4 COVO zu sehen, der den Vollzug den Kantonen überträgt. Auch diese Übertragung wäre nicht notwendig, da die Kantone ohnehin auch für den Vollzug der vom Bund angeordneten Massnahmen zuständig sind (Art. 102 Abs. $2 \mathrm{EpV}^{9}$ ). Die Verordnung trat gemäss Art. 5 am 28. Februar 2020 um $10 \mathrm{Uhr}$ in Kraft, also zwar nach Publikation in der amtlichen Sammlung, aber noch vor der Me-

6 https://www.bag.admin.ch/bag/de/home/krankheiten/ausbrueche-epidemien-pandemien/ aktuelle-ausbrueche-epidemien/novel-cov.html\#-240056265 (zuletzt besucht am 8. März 2020).

7 https://www.bag.admin.ch/bag/de/home/krankheiten/ausbrueche-epidemien-pandemien/ aktuelle-ausbrueche-epidemien/novel-cov.html\#-240056265 (zuletzt besucht am 8. März 2020).

8 Bundesgesetz über die Bekämpfung übertragbarer Krankheiten des Menschen vom 28. September 2012 (EpG; SR 818.101); zum Zweck Gächter/Rütsche (Fn. 4), Rn. 784.

9 Verordnung über die Bekämpfung übertragbarer Krankheiten des Menschen vom 29. April 2015 (EpV; SR 818.101.1). 
dienkonferenz des Bundes (und der entsprechenden Sperrfrist ${ }^{10}$ ). Die Verordnung ist bis zum 15. März 2020 befristet, was die identische Befristung des Versammlungsverbots redundant erscheinen lässt.

\subsection{Rechtsgrundlage}

[8] Die COVO stützt sich auf Art. 6 Abs. 2 Bst. b EpG, der den Bundesrat ermächtigt, in besonderen Lagen Massnahmen gegenüber der Bevölkerung zu treffen. Das Epidemiengesetz des Bundes unterscheidet zwischen normalen, besonderen und ausserordentlichen Lagen. ${ }^{11}$ Zwar kommt dem Bund immer eine Leit- und Koordinationsfunktion $\mathrm{zu}^{12}$ doch sind in normalen Lagen für konkrete Massnahmen die Kantone zuständig (Art. $30 \mathrm{ff}$. EpG); sie haben darüber dem Bund nur Meldung zu erstatten (Art. $13 \mathrm{EpV}$ ). Erst wenn eine besondere Lage auftritt, wird der Bund und für ihn der Bundesrat - zur Anordnung der nämlichen Massnahmen zuständig (Art. 6 Abs. 2 EpG). Die «höchste Alarmstufe» ist die ausserordentliche Lage gemäss Art. 7 EpG, in der dem Bundesrat die Notkompetenz zum Erlass noch weiterer Massnahmen zukommt. Alle Massnahmen werden aber grundsätzlich von den Kantonen vollzogen. ${ }^{13}$

[9] Damit also der Bundesrat zum Erlass der COVO kompetent ist, muss eine besondere Lage vorliegen. Es müssen mithin die Voraussetzungen von Art. 6 Abs. 1 EpG erfüllt sein. Dies ist zunächst der Fall, wenn die WHO eine «gesundheitliche Notlage internationaler Tragweite» festgestellt hat und diese auch die Schweiz betrifft; so geschehen am 30. Januar 2020. ${ }^{14}$ Darüber hinaus ist eine besondere Lage auch dann gegeben, wenn eine Notlage die Kraft der ordentlichen Vollzugsorgane übersteigt (paradoxerweise wird dann aber nicht der Vollzug, sondern nur die Anordnungskompetenz übertragen) und alternativ eine erhöhte Ansteckungs- oder Ausbreitungsgefahr ${ }^{15}$ oder aber eine besondere Gefährdung der öffentlichen Gesundheit vorliegt. ${ }^{16}$ Auch diese Kriterien wären erfüllt. Der Bundesrat war zum Erlass der COVO also kompetent.

[10] Würde sich die Lage dramatisch verschlimmern, wäre denkbar, dass eine ausserordentliche Lage eintritt. Im Gegensatz zu den abschliessenden Massnahmen der Art. 30 ff. EpG, die in normalen und besonderen Lagen den Kantonen beziehungsweise dem Bundesrat zur Verfügung stehen, ist der Kreis der Massnahmen in den ausserordentlichen Lagen nicht beschränkt. Dies entspricht der Konzeption, dass es sich bei Art. 7 EpG um eine blosse deklaratorische Wiederholung der ohnehin bestehenden Notverordnungskompetenz des Bundesrates gemäss Art. 185 Abs. 3

10 Kritik sei an dieser Stelle angebracht an der Verhängung einer «Sperrfrist» für Medienschaffende bis um 10:15 Uhr - also 15 Minuten nach Inkrafttreten der Verordnung. Eine solche Sperrfrist erscheint übertrieben und darf Medien nicht daran hindern, über eine inkraftgetretene Verordnung zu berichten.

11 Thomas Gächter/Sara Lòpez, Entwurf zu einer Totalrevision des Epidemiengesetzes, Sicherheit \& Recht 2011, S. 118 ff., S. 119 f.; Rechsteiner (Fn. 2), Rn. 884.

12 Art. 4 f. EpG; Botschaft zur Revision des Bundesgesetzes über die Bekämpfung übertragbarer Krankheiten des Menschen (Epidemiengesetz, EpG) vom 3. Dezember 2010, BBl 2011311 ff., S. 336.; GäCHTER/RüTsCHE (Fn. 4), Rn. 791.

13 Art. 75 EpG; Botschaft EpG (Fn. 12), S. 365.

14 https://www.who.int/dg/speeches/detail/who-director-general-s-statement-on-ihr-emergency-committee-onnovel-coronavirus-(2019-ncov) (zuletzt besucht am 8. März 2020).

15 Kritisch zum Kriterium Gächter/LòPez (Fn. 11), S. 119.

16 Botschaft EpG (Fn. 12), S. 364; Gächter/Rütsche (Fn. 4), Rn. 793. 
BV $^{17}$ handelt. ${ }^{18}$ Solche Massnahmen können sehr vielfältig sein, da sich die ausserordentlichen Lagen stark unterscheiden können. ${ }^{19}$ Aus dem gleichen Grund wurde bewusst auf eine Umschreibung der Voraussetzungen einer ausserordentlichen Lage verzichtet. ${ }^{20}$ Beispielhaft bestehe eine ausserordentliche Lage etwa bei einer «Bedrohung der äusseren und inneren Sicherheit» durch eine fatale Pandemie wie etwa die Spanische Grippe im Jahr 1918. ${ }^{21}$ Diese Unbestimmtheit ist zwar sachlich überzeugend, rechtsstaatlich jedoch problematisch, da Art. 7 EpG deswegen sowohl bezüglich der Tatbestands- wie auch der Rechtsfolgeseite hochgradig unbestimmt bleibt. Daher ist nur sehr zurückhaltend von einer ausserordentlichen Lage auszugehen. ${ }^{22}$

\section{Veranstaltungen mit mehr als 1000 Personen}

\subsection{Rechtsnatur}

[11] Nach der Formulierung von Art. 2 Abs. 1 COVO sind sämtliche Veranstaltungen mit mehr als 1000 Personen verboten. Der Wortlaut spricht also für ein striktes Verbot ohne Möglichkeit zur Erteilung einer Ausnahmebewilligung. Sachlich nachvollziehbar ist, dass eine strikte Handhabung der Reglung gewünscht ist, um eine Umgehung zu verhindern. Gleichwohl steht das Verbot im Widerspruch zum Verhältnismässigkeitsgebot, ${ }^{23}$ wenn es eine Einzelfallbetrachtung durch die Vollzugsbehörden a priori ausschliesst. So wird auch die - durch Art. 22 BV immerhin grundrechtlich geschützte - Durchführung von Versammlungen verboten, bei denen eine Verbreitung des Coronavirus ausgeschlossen wäre.

[12] Insbesondere in Anbetracht der - notgedrungen - arbiträren Grenze von 1000 Personen steht die fehlende Möglichkeit zur Erteilung einer Ausnahmebewilligung auch im Widerspruch zum Rechtsgleichheitsgebot ${ }^{24}$, da im Ergebnis durch Art. 2 Abs. 1 COVO Gleiches ungleich behandelt wird (eine Veranstaltung mit 995 Personen und eine Veranstaltung mit 1005 Personen). Dies ist zwar ein notwendiger Effekt jeder Grenze, er könnte durch die Möglichkeit zur Erteilung einer Ausnahmebewilligung aber behoben werden. Auch der diesbezüglich einschlägige ${ }^{25}$ Art. 40 EpG verlangt, die gesellschaftlichen und wirtschaftlichen Folgen der Anordnung zu berücksichtigen. ${ }^{26}$ Dies spricht für eine verfassungskonforme Auslegung, die Ausnahmen durch die zuständigen Vollzugsbehörden erlaubt. Da es sich hierbei um eine Ausnahme von einem in der COVO abschliessend definierten Verbot handelt, wäre sinnvollerweise die Bewilligungsbehörde das BAG. ${ }^{27}$ Diese Zuständigkeit würde gleichzeitig die mit dem übrigen Vollzug befassten kan-

17 Bundesverfassung der Schweizerischen Eidgenossenschaft vom 18. April 1999 (BV; SR 101).

18 Botschaft EpG (Fn. 12), 365; vgl. aber Giovanni BiagGini, Kommentar zur Bundesverfassung, 2. Aufl., Zürich 2017, Art. 185 Rn. 12a; zum alten Recht BGE 131 II 670 E. 3.1 S. 676 f.

19 Dazu Urs SAxer, in: St. Galler Kommentar zur Bundesverfassung, 3. Aufl., Zürich/St. Gallen 2014 (zit. SGK BVVerfasser), Art. 185 Rn. 89.

20 Botschaft EpG (Fn. 12), S. 366; Rechsteiner (Fn. 2) Rn. 884 f.

21 Botschaft EpG (Fn. 12), S. 363.

22 GäCHTER/Rütsche (Fn. 4), Rn. 794.

23 Art. 5 Abs. 2 BV.

24 Art. 8 Abs. 1 BV.

25 Botschaft EpG (Fn. 12), S. 365.

26 Botschaft EpG (Fn. 12), S. 392.

27 Der Bund kann auch Elemente des Vollzugs an sich ziehen (vgl. Art. 102 Abs. 2 EpV). 
tonalen Stellen entlasten und eine einheitliche Praxis der Ausnahmen sicherstellen. Zudem sind die kantonalen Behörden unter Umständen erheblichem politischem Druck ausgesetzt; demgegenüber könnte das BAG eher eine restriktive Anwendung der Ausnahmepraxis ${ }^{28}$ sicherstellen.

[13] Das Verbot richtet sich deswegen gegen Veranstaltungen, weil dort das Risiko der Verbreitung des Coronavirus im Vergleich zum gewöhnlichen (Berufs-)Alltag erhöht ist. ${ }^{29}$ Damit eine Veranstaltung zur Kandidatin für eine Ausnahmebewilligung werden kann, müsste hinreichend überzeugend dargetan sein, dass durch die Natur der Veranstaltung oder durch besondere Vorkehren das Verbreitungsrisiko auf das Niveau einer alltäglichen Tätigkeit reduziert werden konnte. Durch eine Ausnahme dürfen die Vollzugsbehörden aber den Zweck der COVO nicht unterlaufen. Dieser Zweck ist nach Art. 1 Abs. 2 COVO dreigliedrig: ${ }^{30}$

[14] Erstens soll die Verbreitung verhindert bzw. eingedämmt werden. Dementsprechend müsste mit hinreichender Sicherheit ausgeschlossen werden können, dass ansteckende Personen an einer zu bewilligenden Veranstaltung teilnehmen. Dies ist beim - bereits vor Ausbruch der CoronavirusKrankheit ansteckenden - Coronavirus schwierig, da gerade nicht auf die Symptome abgestellt werden kann. Es dürfen an einer Veranstaltung demnach keine Personen teilnehmen, die nach den jeweils aktuellen wissenschaftlichen Erkenntnissen eine erhöhte Krankheitswahrscheinlichkeit aufweisen. Auszuschliessen wären zum gegenwärtigen Zeitpunkt etwa Personen, die in betroffenen Gebieten waren oder Kontakt zu solchen Personen hatten.

[15] Zweitens sollen besonders vulnerable Personen geschützt werden. Eine Veranstaltung müsste demnach ohne Personen stattfinden, die ein besonderes Ansteckungsrisiko aufweisen oder bei denen bei einer Ansteckung mit einem gravierenden Krankheitsverlauf zu rechnen ist. Dies sind nach heutigem Kenntnisstand insbesondere ältere oder vorbelastete Personen.

[16] Drittens sollen Übertragungsketten unterbrochen werden, was bedingt, dass potentiell neu infizierte Personen rasch eruiert, informiert und unter Quarantäne gestellt werden können. Die anwesenden Personen müssen demnach namentlich bekannt und innert nützlicher Frist kontaktierbar sein. Dies dürfte bei Veranstaltungen mit mehr als 1000 Personen ausgesprochen schwierig sein. Darüber hinaus nimmt bei einer grösseren Anzahl zu kontaktierender Personen auch die Kontaktaufnahme selbst länger in Anspruch.

[17] Zwar mutet die Ausnahmemöglichkeit eher theoretisch an, da unter diesen Umständen schwer vorstellbar ist, welche Veranstaltung mit mehr als 1000 Personen bewilligt werden könnte. Dennoch ist nicht auszuschliessen, dass einer Veranstalterin oder einem Veranstalter der Nachweis gelingen könnte (beispielsweise weil alle anwesenden Personen keinen Aussenkontakt hatten). Diesfalls wäre eine Bewilligung zu erteilen und eben gerade nicht nur mit Verweis auf die fehlende Ausnahmemöglichkeit ${ }^{31}$ zu verweigern.

[18] Das Veranstaltungsverbot des Art. 2 Abs. 1 COVO gilt direkt aufgrund der Verordnung selbst. Es muss weder angeordnet noch festgestellt werden.

28 Dazu Ulrich Häfelin/Georg Müller/Felix Uhlmann, Allgemeines Verwaltungsrecht, 7. Aufl., Zürich/St. Gallen 2016, Rn. 2674.

29 Kriterien bezüglich Vollzug Veranstaltungsverbot vom 28. Februar 2020, abrufbar unter https://www. bag.admin.ch/dam/bag/de/dokumente/mt/k-und-i/aktuelle-ausbrueche-pandemien/2019-nCoV/ kriterien-veranstaltungen-kantone.pdf.download.pdf/kriterien_veranstaltungen_kantone_04_03_2020.pdf (zuletzt besucht am 8. März 2020), Ziff. 1.

30 Allgemein GÄCHTER/RüTsche (Fn. 4), Rn. 776.

31 HäFelin/MülleR/UhlmanN (Fn. 28), Rn. 2666. 


\subsection{Erfasste Veranstaltungen}

[19] In der deutschen Fassung der COVO besteht eine Unklarheit bezüglich der erfassten Veranstaltungen. Art. 2 Abs. 1 COVO spricht von Veranstaltungen, an denen sich «Personen aufhalten». Demgegenüber nennt Art. 2 Abs. 2 COVO Personen, die «teilnehmen». Zumindest nach einem engen Wortverständnis sind wenigstens die Veranstalterinnen und Veranstalter eben gerade keine «Teilnehmer». Es ist aber aus mehreren Gründen nicht von diesem engen Wortverständnis auszugehen, sondern vom Verständnis von Abs. 1. Dafür spricht zunächst die französische Fassung, die in beiden Fällen wortgleich von accueillir spricht. Auch sachliche Überlegungen sprechen für dieses Verständnis, da die Übertragbarkeit des Coronavirus nicht auf die Rolle einer Person Rücksicht nimmt. Gemeint sind also Veranstaltungen, bei denen 1000 Personen insgesamt anwesend sind. Service-, Sicherheits-, Reinigungs- und sonstiges Personal, Veranstalterinnen und Veranstalter, Musikerinnen und Musiker, Rednerinnen und Redner, Zaun- und Zufallsgäste usw. zählen demnach ebenfalls zu dieser Zahl, nicht bloss Gäste. Eine Veranstaltung mit genau 1000 Personen ist nach dem Wortlaut von Art. 2 Abs. 1 COVO aber noch zulässig. ${ }^{32}$

[20] Unerheblich ist auch, ob die Anzahl geplant oder zufällig überschritten wurde. Es liegt in der Verantwortung der Veranstalterinnen und Veranstalter, abzuschätzen, ob die Anzahl erreicht wird oder nicht. Werden 1000 Personen an einer Veranstaltung erreicht, ist sie abzusagen oder abzubrechen. Insbesondere mit dem Verbotszweck unvereinbar dürfte demgegenüber sein, eine Eintrittskontrolle vorzusehen, welche die gleichzeitig Teilnehmenden auf unter 1000 beschränkt. Bei diesem Vorgehen bilden sich nämlich aller Erwartung nach Schlangen von Personen, die eine erneute Zutrittsmöglichkeit abwarten. Da sich solche Personen ebenfalls am Veranstaltungsort aufhalten, müssen sie ebenfalls gezählt werden und führen dazu, dass die Veranstaltung verboten ist. Zulässig wäre dagegen, die überzähligen Personen nach Hause zu schicken und die Türen zu schliessen, um damit eine Schlangenbildung zu verhindern.

[21] Neben dem Begriff der anwesenden Personen ist auch unklar, was als «Veranstaltung» zählen soll - ob etwa Büros, Sitzungen, Züge, Restaurants oder Einkaufscenter erfasst werden. Der Begriff wird im Bundesrecht verwendet, aber soweit ersichtlich nicht definiert. ${ }^{33}$ Hier hilft einerseits eine Betrachtung des Art. 40 EpG. Neben den Veranstaltungen des Art. 40 Abs. 2 Bst. a EpG nennt Bst. b «Schulen, andere öffentliche Institutionen und private Unternehmen». Damit grenzt der Gesetzgeber den Begriff der Veranstaltung ab von den Schulen, öffentlichen Institutionen, privaten Unternehmen oder Gebäuden. Das Verhältnismässigkeitsgebot verlangt zudem, dass alltägliche Besorgungen nach Möglichkeit nicht vom Verbot erfasst sind. Demnach wäre ein vorbestimmtes oder spontanes, über bloss zufällige Anwesenheit hinausgehendes, Programm für mehrere Personen notwendig, um von einer Veranstaltung zu sprechen. Diese Anhaltspunkte lassen zumindest eine gewisse Konkretisierung zu, die aber wohl nicht ohne eine Betrachtung im Einzelfall auskommt. Allgemein bezeichnet der Begriff demnach über die Besorgung des Lebensalltags hinausgehende Versammlungen. ${ }^{34}$

32 Ebenso Kriterien (Fn. 29), Ziff. 3.

33 Eine Definition mit Beispielen liefert das BAG in den Kriterien (Fn. 29), Ziff. 2. Abgesehen vom Kriterium der räumlichen und zeitlichen Begrenzung (das wenig abgrenzungskräftig ist, da letztlich alles räumlich und zeitlich begrenzt ist) entspricht diese Definition der hier gegebenen.

34 Dies entspricht im Ergebnis der Empfehlung auf https://www.bag.admin.ch/bag/de/home/krankheiten/ ausbrueche-epidemien-pandemien/aktuelle-ausbrueche-epidemien/novel-cov/massnahmen-des-bundes.html (zuletzt besucht am 8. März 2020). 


\subsection{Verletzungen}

[22] Die Rechtsfolgen einer Verletzung von Art. 2 Abs. 1 COVO richten sich in erster Linie nach dem Verordnungszweck. Primär fallen daher Anordnungen nach Art. 3 Abs. 2 COVO in Betracht, allen voran natürlich das vorgängige Verbot oder - im Falle einer bereits bestehenden Veranstaltung - die nachträgliche Auflösung der Veranstaltung. Art. 3 Abs. 2 COVO ist aber zu unbestimmt, um Rechtsgrundlage für konkrete Massnahmen zu sein, die sich nicht (wie eben das Verbot oder die Auflösung einer Versammlung) aus dem Rest der COVO ergeben. Die zuständigen Behörden können aber Massnahmen gegen Personen nach den Art. 33 ff. EpG anordnen. ${ }^{35}$ Sie können Massnahmen auch auf eine andere Rechtsgrundlage, etwa aus dem Polizei- oder Gesundheitsrecht, oder die polizeiliche Generalklausel stützen, wo die entsprechenden Anforderungen erfüllt sind.

[23] Als Rechtsgrundlage für Strafen eignet sich eine Verordnung zwar nicht (Art. 1 StGB ${ }^{36}$ ), doch enthalten Art. 82 f. EpG Strafbestimmungen. Demnach ist es eine Übertretung, sich einer Massnahme wie der vorliegenden zu widersetzen, und wird mit Busse bestraft. ${ }^{37}$ Bei besonders qualifiziertem Vorsatz, nämlich bei «gemeiner Gesinnung», kann zudem eine Freiheitsstrafe nach Art. 231 StGB verhängt werden.

\section{Veranstaltungen mit weniger als 1000 Personen}

\subsection{Rechtsnatur}

[24] Bei Veranstaltungen mit weniger als 1000 anwesenden Personen verlangt Art. 2 Abs. 2 COVO, dass «die Veranstalter zusammen mit der zuständigen kantonalen Behörde eine Risikoabwägung vornehmen, ob sie die Veranstaltung durchführen können oder nicht.» Aus dem Wortlaut ist nicht völlig klar, ob es sich dabei um ein Verbot mit Bewilligungsvorbehalt, eine Erlaubnis mit Verbotsvorbehalt oder eine blosse Meldepflicht handelt.

[25] Verschiedenes spricht dafür, dass Art. 2 Abs. 2 COVO eine bundesrechtliche Meldepflicht ${ }^{38}$ ist. So erklärt das Bundesamt für Gesundheit auf seiner Webseite, der Bundesrat habe absichtlich entschieden, dass die Kantone entscheiden können, Veranstaltungen mit weniger als 1000 Personen «zu verbieten». ${ }^{39}$ Dies spricht gegen ein direkt anwendbares Verbot mit Bewilligungsvorbehalt. Auch in sachlicher Hinsicht wäre ein solches Verbot nicht angebracht, zumal die COVO keine Untergrenze an teilnehmenden Personen vorsieht. Betroffen wären also ausserordentlich viele Veranstaltungen, die alle einer Bewilligung bedürften. Die kantonalen Behörden würden durch die Anzahl an Bewilligungsgesuchen überlastet. ${ }^{40}$ Sinnvoller ist demnach, bei Art. 2 Abs. 2

35 Dazu Gächter/Rütsche (Fn. 4), Rn. 798.

36 Schweizerisches Strafgesetzbuch vom 21. Dezember 1937 (StGB; SR 311.0).

37 Art. 83 Abs. 1 Bst. j EpG.

38 Die allerdings von den Meldepflichten nach EpG zu unterscheiden ist.

39 https://www.bag.admin.ch/bag/de/home/krankheiten/ausbrueche-epidemien-pandemien/ aktuelle-ausbrueche-epidemien/novel-cov/massnahmen-des-bundes.html (zuletzt besucht am 8. März 2020).

40

Vgl. etwa zum Kanton Aargau, der eine Untergrenze von immerhin 150 Personen eingeführt hat, «Aargauer Zeitung» vom 1. März 2020, https://www.aargauerzeitung.ch/aargau/kanton-aargau/corona-virus-aargauerveranstalter-reichen-fast-400-gesuche-innert-eines-tages-ein-eines-wird-abgelehnt-136442062 (zuletzt besucht am 8. März 2020). 
COVO von einer direkt anwendbaren Meldepflicht auszugehen. Gestützt darauf wird den Kantonen ermöglicht, nötigenfalls ein Verbot oder sonstige Einschränkungen anzuordnen. Diese Anordnungen stützen sich aber nicht auf die COVO, sondern die gesetzliche Kompetenz der Kantone, Veranstaltungen zu verbieten oder einzuschränken (Art. 40 Abs. 2 Bst. a EpG). Selbstverständlich sind auch hierbei die grund- und verwaltungsrechtlichen Anforderungen einzuhalten, insbesondere das Störerprinzip.

\subsection{Erfasste Veranstaltungen}

[26] Wie bereits ausgeführt sieht der Wortlaut von Art. 2 Abs. 2 COVO keine Untergrenze für erfasste Veranstaltungen vor und grenzt die Veranstaltungen auch sachlich nicht ein. Vor dem Hintergrund möglicher Veranstaltungen ist dies als zu pauschal abzulehnen: So werden Altersgymnastik, Dorffasnacht, Kaffichränzli, Sonntagsgottesdienst, Swingerclub und Theatervorführung alle gleich behandelt. Auch hier ist, wiederum vor dem Hintergrund der Zweckbestimmung des Veranstaltungsverbots, ein verfassungskonformer differenzierter Auslegungs- beziehungsweise Anwendungsvorschlag anzubringen. Gemäss Botschaft ist das Veranstaltungsverbot in Art. 40 EpG «auf die kollektive Ebene ausgerichtet (social distancing)», "da Menschenansammlungen für die Ausbreitung bestimmter Krankheiten (z. B. Grippe oder Masern) besonders förderlich sind.» ${ }^{41}$ Notwendig ist damit eine eigentliche «Menschenansammlung», was zumindest mehr als einige wenige Personen sind. Eine solche Untergrenze ist - genauso wie die Grenze bei 1000 Personen - letztlich arbiträr, doch ist es sinnvoller, wenn sie im Interesse der Rechtssicherheit ausformuliert ist. Im Sinne eines «seuchenrechtlichen Veranstaltungsbegriffs» sollte etwa das Zusammenkommen von 30 oder weniger Personen deshalb grundsätzlich nicht als Veranstaltung gelten und daher nicht unter die Meldepflicht fallen, solange keine besonderen Risiken bestehen. Gleich wie oben (Ziff. 3.1.) beurteilt sich das Vorliegen eines besonderen Risikos nach den Zwecken der COVO. Da unter der Grenze aber keinerlei Abwägung stattfinden kann (die Vollzugsorgane wissen ja von der Versammlung mangels Meldepflicht nichts), scheint die Zahl von 150 Personen ${ }^{42}$ eher hoch, wenn auch zur Entlastung des Vollzugs nachvollziehbar. Selbstverständlich sollen die vorstehenden Überlegungen nicht die Veranstalterinnen und Veranstalter davon entbinden, durch geeignete Vorkehrungen und Information der Teilnehmenden das Ihre zur Eindämmung des Coronavirus beizutragen.

[27] Oberhalb dieser Grenze ist nach dem Gesagten von einer Versammlung und damit von einer Meldepflicht auszugehen. Die meisten Kantone haben ein Formular oder eine Hotline eingerichtet, mit dem der Kontakt zur zuständigen Behörde aufgenommen wird. ${ }^{43}$ Gestützt auf die Meldung muss der Kanton mit der Veranstalterin oder dem Veranstalter gemäss Art. 2 Abs. 2 COVO eine «Risikoabwägung» vornehmen. Auch dabei müssen spezifisch die Zwecke aus Art. 1 Abs. 2 COVO als Leitlinie gelten. Naturgemäss fällt dabei neben den geplanten Aktivitäten und den Räumlichkeiten insbesondere auch die Anzahl der Teilnehmenden ins Gewicht, so dass ein

41 Botschaft EpG (Fn. 12), 392.

42 Kriterien (Fn. 29), Ziff. 4.

43 Z.B. für den Aargau https://www.ag.ch/de/themen_1/coronavirus_2/kontaktformular_coronavirus/ kontaktformular_coronavirus.jsp oder für Zürich 0800044117. 
Verbot einer Veranstaltung umso eher in Betracht fällt, je näher sie der 1000-Personen-Grenze des Art. 2 Abs. 1 COVO kommt. ${ }^{44}$

[28] Unbefriedigend ist die grosse Verschiedenheit der kantonalen Regelungen in diesem Bereich. ${ }^{45}$ Die Ausbreitung eines Virus ändert sich nicht an der kantonalen Grenze, weshalb kein Grund für föderalistisch begründete Ungleichbehandlungen besteht. Auch sprechen Gründe der Rechtssicherheit dafür, dass die Kantone sich auf eine gemeinsame Regelung einigen oder der Bund eine solche trifft. Zwar hat das BAG am vergangenen Mittwoch, 4. März 2020, im Sinne einer Hilfestellung für die Kantone ein Dokument mit Kriterien ${ }^{46}$ für den Vollzug der Verordnung aufgestellt, doch sind die Regelungen nach wie vor sehr unterschiedlich. Es wäre zu hoffen, dass sich, auch zusammen mit dem vorstehend gemachten Auslegungsvorschlag, eine Angleichung einstellt. Ein Teil der unterschiedlichen Regelungen ist zudem als Ermessensunterschreitung durch die Kantone zu qualifizieren. Nach dem Wortlaut von Art. 2 Abs. 2 COVO ist eine Risikoabwägung bei Veranstaltungen unter 1000 Personen zwingend. Wenn Veranstaltungen ab einer bestimmten Grösse pauschal verboten werden, ohne dass eine Risikoabwägung im Einzelfall stattfindet, unterschreiten die Kantone das von der COVO eingeräumte Interesse. Das Gleiche gilt bei der pauschalen Erlaubnis für Veranstaltungen bis zu einer bestimmten Anzahl Personen, soweit nicht - wie etwa vorstehend - der COVO durch Auslegung des Veranstaltungsbegriffs eine implizite Untergrenze entnommen wird. Erst ab 150 Personen von einer Veranstaltung auszugehen, strapaziert die Auslegung allerdings. Aus Gründen der Normbestimmtheit wäre eine so hohe Grenze in der Verordnung zu nennen.

\subsection{Rechtsfolgen}

[29] Gemäss Art. 3 Abs. 1 COVO haben die kantonalen Vollzugsorgane relativ weitgehende Zutritts- und Kontrollrechte, die sie einsetzen können, um die Einhaltung der COVO zu überwachen. Dass diese Rechte im Verhältnis zu den verbotenen Veranstaltungen mit mehr als 1000 Personen bestehen, versteht sich von selbst, da dort die Behörden sicherstellen müssen, dass die Veranstaltung nicht stattfindet. Diese Rechte dürften den Behörden aber auch im Verhältnis zu allen anderen Veranstaltungen zukommen. Dies ergibt sich aus Art. 3 Abs. 2 COVO: Wären nur verbotene Veranstaltungen der Kontrollpflicht unterworfen, wären nicht unbestimmte Anordnungsmöglichkeiten die Handhabe der Behörde, sondern eben nur die Auflösung der Veranstaltung. Dies wäre für zulässige Veranstaltungen unverhältnismässig. Daher sind die Kontrollbefugnisse und als Folge anlässlich einer Kontrolle festgestellter Mängel auch die Anordnungsmöglichkeit der Behörden in Art. 3 COVO für alle Veranstaltungen, unabhängig von der Anzahl anwesender Personen, einschlägig.

\section{Rechtsschutz}

[30] Die Rechtsweggarantie (Art. 29a BV) verlangt bei Rechtsstreitigkeiten die Möglichkeit, an ein Gericht zu gelangen. Die Versammlungsverbote der COVO fallen (wie weitere denkbare Mass-

\footnotetext{
44 Ebenso Kriterien (Fn. 29), Ziff. 4.

45 Vgl. dazu etwa «St. Galler Tagblatt» vom 2. März 2020, S. 2.

46 Kriterien (Fn. 29).
} 
nahmen) zweifellos in diese Kategorie. Ein Verbot wie das vorliegende tangiert verschiedene Grundrechte, insbesondere die Bewegungsfreiheit (Art. 10 Abs. 2 BV), die Versammlungsfreiheit (Art. $22 \mathrm{BV}$ ) und die Wirtschaftsfreiheit Art. $27 \mathrm{BV}$ ). ${ }^{47}$ Auch in besonderen und ausserordentlichen Lagen müssen die Grundrechte gewahrt werden, was bedeutet, dass Eingriffe nur unter Einhaltung der Schranken des Art. 36 BV zulässig sind.$^{48}$ Diese Fragen müssen gerichtlich überprüft werden können (notfalls auch nachträglich), da sie grundsätzlicher Natur sind.$^{49}$ Die Formulierung in Art. 3 Abs. 2 COVO, es seien die "Anordnungen der Kantone unverzüglich umzusetzen», kann als implizite Entziehung der aufschiebenden Wirkung einer Beschwerde gedeutet werden. Dies ist zur Sicherung des Verordnungszwecks sinnvoll. Da eine entsprechende Anordnung im Gesetz fehlt und auf Verordnungsstufe nicht implementiert werden kann, ist aber klar, dass die Wiedererteilung der aufschiebenden Wirkung durch die Rechtsmittelbehörden möglich ist.

[31] Da das Versammlungsverbot für Versammlungen mit mehr als 1000 Personen direkt aufgrund der COVO gilt, fehlt es an einem Anfechtungsobjekt; die zuständige kantonale Behörde wird daher in solchen Fällen anfechtbare Feststellungsverfügungen zu erlassen haben. Andere Anordnungen der Kantone sind ebenfalls anfechtbar. Der Rechtsweg gegen diese Anordnungen der Kantone ist unproblematisch: Es handelt sich um den "gewöhnlichen» kantonalen Rechtsweg, auch soweit die Rechtsgrundlage eine bundesrätliche Verordnung ist. Unklar ist der Rechtsweg demgegenüber bei der Anfechtung der (kantonalen) Feststellungsverfügung über das bundesrechtliche, direkt gemäss COVO geltende Verbot. Den kantonalen Verwaltungsgerichten wäre die Aufhebung des bundesrechtlichen Verbots verwehrt. Daher wäre eine Anfechtbarkeit beim Bundesverwaltungsgericht sinnvoller, was aber zu unterschiedlichen Rechtswegen und mitunter zu schwierigen Abgrenzungsfragen führen würde (zum Beispiel bei einer Veranstaltung, die eine kantonale Einschränkung erfährt, dann aber wegen Überschreiten der Personengrenze unter Art. 2 Abs. 1 COVO fällt und verboten ist).

[32] Nach Art. 63 EpG besteht eine Ausfallhaftung, ${ }^{50}$ die aber nur bei Massnahmen gegen Einzelpersonen anwendbar ist. Ansprüche gegen Anordnungen der COVO sind daher nur gestützt auf die allgemeine Staatshaftung denkbar. Ein solcher Haftungsanspruch dürfte aber am Kriterium der Widerrechtlichkeit scheitern. ${ }^{51}$

\section{Fazit}

[33] Schon die Römer kannten das Sprichwort «In der Not schweigen die Gesetze»; zweifellos können Notsituationen Einschränkungen, teils empfindlicher Art, erfordern. Ganz zum Schweigen gebracht werden dürfen die rechtsstaatlichen Erfordernisse aber nicht - im Gegensatz zum römischen Vorbild unseres Notrechts. Auch in ausserordentlichen Lagen gelten Legalitätsprinzip, Verhältnismässigkeitsgebot und Grundrechte. ${ }^{52}$ Diese Tatsache ist bei der Anwendung und Auslegung der Verordnung zu berücksichtigen. Wie der vorliegende Text gezeigt hat, kann so

GÄCHTER/Rütsche (Fn. 4), Rn. 780.

48 Rechsteiner (Fn. 2), Rn. 891.

49 BGE 131 II 670 E. 3.1 S. $676 \mathrm{f}$.

50 Zur Ausfallhaftung Urteil des Bundesgerichts 2A.462/2002 vom 26. Mai 2003 E. 4.

51 Zur Haftung Rechsteiner (Fn. 2), Rn. 890 u. 948 ff.

52 Rechsteiner (Fn. 2), Rn. 891. 
eine Handhabe gefunden werden, welche rechtsstaatlichen Bedenken Rechnung trägt, ohne die Effektivität der Verordnung einzuschränken.

Benjamin MärkLı ist Tutor für Bundesstaatsrecht an der Universität St. Gallen und Mitarbeiter am Lehrstuhl von Prof. Benjamin Schindler.

Ich danke B.A. Sven Infanger, der nicht nur den Anstoss zu diesem Artikel gegeben hat, sondern durch wertvolle Recherchen und inhaltliche Hinweise die zeitgerechte Fertigstellung des Artikels überhaupt erst ermöglichte. 